\title{
Monte Carlo Simulation Using Moments of Random Variables
}

\author{
Yan-Gang Zhao ${ }^{1}$, Tetsuro Ono ${ }^{2}$ and Kiyoshi Ishii ${ }^{3}$ \\ ${ }^{1}$ Associate Professor, Nagoya Institute of Technology, \\ Gokiso-cho, Shyowa-ku, Nagoya 466-8555 Japan (yzhao@archi.ace.nitech.ac.jp) \\ ${ }^{2}$ Professor, Nagoya Institute of Technology (ono@archi.ace.nitech.ac.jp) \\ ${ }^{3}$ Chief Research Engineer, Shimizu Corporation (kishii@sit.shimz.co.jp)
}

\begin{abstract}
In the present study, in order to include the random variables with an unknown cumulative distribution function (CDF) into Monte-Carlo Simulation, an inverse normal transformation is suggested. The random variables with an unknown CDF are expressed as a simple function of a standard normal random variable, and the function is determined using the first few statistical moments which are generally available from the statistical data of the random variables. Using the proposed method, the random numbers of random variables with an unknown CDF can be easily generated utilizing those of a standard normal random variable, which is generally considered to be quite easily generated. Some examples are presented from which the efficiency of the method is investigated. It is found that although the method is quite simple, it is accurate enough to include the random variables with unknown CDF in the Monte-Carlo Simulation for structural reliability.
\end{abstract}

Keywords: Structural reliability ; Monte-Carlo Simulation ; probability distribution ; random number generation ; statistical moment

\section{Introduction}

In Monte-Carlo Simulation (MCS) of structural reliability, the basic random variables which represent uncertain quantities, such as loads, environmental factors, material properties, and structural dimensions are generally assumed to have a known cumulative distribution function (CDF) or probability density function (PDF), and the generation of random numbers of these random variables is generally conducted based on the CDF of the random variables. In reality however, the PDF/CDF of some basic random variables are often unknown and the probabilistic characteristics of these variables are often expressed using statistical moments only. Under such a condition, the MCS cannot be applied and a strict evaluation of the probability of failure is not possible. Thus, an alternative measure is required.

In the present study, in order to include the random variables with unknown CDFs into MCS, an inverse normal transformation is suggested. The random variables with an unknown CDF are expressed as a simple function of a standard normal random variable, and the function is determined using the first few statistical moments which are generally available from the statistical data of the random variables. Using the proposed method, the random numbers of random variables with

Contact Author: Yan-Gang Zhao, Nagoya Inst. of Technology, Gokiso-cho, Showa-ku, Nagoya 466-8555 Japan

Tel: +81-52-735-5200 Fax: +81-52-735-5200

e-mail: yzhao@archi.ace.nitech.ac.jp

(Received September 7, 2001 ; accepted December 17, 2001) an unknown CDF can be easily generated utilizing those of a standard normal random variable, which is generally considered to be quite easily generated.

Some examples are presented from which the efficiency of the method is investigated. It is found that although the method is quite simple, it is accurate enough to include the random variables with unknown CDF in the MCS for structural reliability.

\section{Brief Reviews}

If a random variable $x$ has a known $\mathrm{CDF} F$, then, the random numbers of $x$ used in MCS are generally generated using the following equation:

$$
x=F^{-1}[\Phi(u)]
$$

where $F$ is the CDF of $x$, and $\Phi$ is the CDF of a standard normal random variable $u$.

Eq. 1 is known as the inverse Rosenblatt transformation, here after it is simply referred as to $u-x$ transformation. If $F$ is known, the random numbers of $x$ can be easily generated using those of $u$. In reality however, due to the lack of statistical data, the CDF of some basic random variables are often unknown and the probabilistic characteristics of these variables are often expressed using statistical moments only. Under such a condition, the MCS cannot be applied.

When the CDF of a random variable is unknown, an approach based on the Bayesian notion can be used (Der Kiureghian \& Liu 1986; Der Kiureghian 1989), in which the distribution is assumed to be a weighed average of all candidate distributions. In this type of model- 
ing, the weights represent the subjective probabilities of each candidate distribution being the true distribution, as suggested by Der Kiureghian \& Liu (1986). For a variable $x_{1}$ with $k$ candidate distributions $F_{1 i}\left(x_{1}\right), i=1$, $\ldots, k$, the Bayesian distribution is written in the form of

$$
F_{1}\left(x_{1}\right)=\sum_{i=1}^{k} p_{1 i} F_{1 i}\left(x_{1}\right)
$$

in which $p_{1 i}$, satisfying $\sum_{i=1}^{k} p_{1 i}=1$, are the weights. Furthermore, all candidate distributions are assumed to have the same mean and variance, because these are assumed to be known quantities.

After obtaining the distribution in Eq. 2, the CDF of $x$ becomes known and the MCS can be conducted using conventional methods. However, the problem arises as to how to select the candidate distributions and the weights.

In order to realize the transformation expressed in Eq. 1 without using the CDF of $x$, a rational and practical approach in this case is to build a direct $u-x$ transformation function using the moment information of $x$. The simplest model is utilizing the second-moment method (Ang \& Cornell 1974; Ang \& Tang 1984). Since the first two moment information is generally inadequate to reflect the other properties such asymmetry and sharpness of the distribution, a high-order moment standardization technique (Ono \& Idota 1986) has been proposed. The method involves the use of the following inverse transformation of Eq. 1:

$$
u=S_{x}(x)=\sum_{j=1}^{k} a_{j} x^{j-1}
$$

where $a_{i}, j=1, \ldots, k$, are deterministic coefficients that are obtained by setting the first $k$ central moments of $S_{x}(x)$ equal to those of $u$.

Using Eq. 3, to obtain the $k$ th moment standardization function, the $k(k-1)$ th central moment of $x$ must be determined. Even for the third-moment standardization, the first six moments of $x$ must be used; and while the fourth-moment standardization, the first 12 moments of $x$ must be determined. As such the standardization becomes complicated, and obtaining the accurate standardization function becomes difficult. In order avoid these shortcomings, a direct $u$ - $x$ transformation is suggested using the polynomial forms of $u$ (Zhao $\&$ Ono 2000). Without loss of generality, for a standardized random variable

$$
x_{s}=\frac{x-\mu}{\sigma}
$$

where $\mu$ and $\sigma$ are the mean value and standard deviation of $x$, respectively, the $u-x$ transformation is built as the following form.

$$
x_{s}=S_{u}(u)=\sum_{j=1}^{k} a_{j} u^{j-1}
$$

where $a_{j}, j=1, \ldots, k$, are deterministic coefficients that are obtained by making the first $k$ central moment of $S_{u}(u)$ to be equal to those of $x_{s}$.

Using Eq. 5, to obtain the $k$ th moment transformation function, only the first $k$ central moments of $x_{s}$ are needed. After obtaining the deterministic coefficients $a_{i}, j=1, \ldots, k$, the random numbers of $x$ can be easily obtained using Eq. 5.

\section{Generation of Random Numbers Using Higher-Or- der Moments}

\section{Random Number Generation Using the First Three Moments}

For a random variable with only the first three moments being known, the $u-x$ transformation is given as

$$
x_{s}=S_{u}(u)=a_{1}+a_{2} u+a_{3} u^{2}
$$

After obtaining the coefficients $a_{1}, a_{2}$ and $a_{3}$, the random number of $x$ can be readily obtained with the aid of the following equation using those of $u$.

$$
x=\sigma\left(a_{1}+a_{2} u+a_{3} u^{2}\right)+\mu
$$

Making the first three central moments of $S_{u}(u)$ equal to those of $x_{s}$, the following equations containing $a_{1}, a_{2}$ and $a_{3}$ are obtained.

$$
\begin{aligned}
& \mu_{s u}=a_{1}+a_{3}=0 \\
& \sigma_{s u}^{2}=a_{2}^{2}+2 a_{3}^{2}=1 \\
& \alpha_{3 s u} \sigma_{s u}^{2}=6 a_{2}^{2} a_{3}+8 a_{3}^{3}=\alpha_{3}
\end{aligned}
$$

where $\alpha_{3 s u}$ and $\alpha_{3}$ are the third dimensionless central moments, i.e. the skewnesses of $S_{u}$ and $x$, respectively.

After simplification, $a_{1}, a_{2}$ and $a_{3}$ are given as (Zhao $\&$ Ono 2000).

$$
\begin{aligned}
& a_{3}=-a_{1}= \pm \sqrt{2} \cos \left[\frac{\pi+|\theta|}{3}\right] \\
& a_{2}=\sqrt{1-2 a_{3}^{2}} \\
& \theta=\arctan \left(\frac{\sqrt{8-\alpha_{3}^{2}}}{\alpha_{3}}\right)
\end{aligned}
$$

The signs in Eq. 9 are taken to be the sign of $\alpha_{3}$.

From Eq. 11, in order to make Eq. 6 operable, $\alpha_{3}$ should be limited in the range of

$$
-2 \sqrt{2} \leq \alpha_{3} \leq 2 \sqrt{2}
$$

From the investigation of the skewnesses of some commonly used random variables, Eq. 6 is found to be generally operable in engineering. It has been shown that Eq. 7 essentially defines a three-parameter distribution that can reflect the skewness of statistical data effectively (Zhao et al 2001).

\section{Random Number Generation Using the First Four Mo- ments}

For a random variable with only the first four moments being known, the $u-x$ transformation is given as:

$$
x_{s}=S_{u}(u)=a_{1}+a_{2} u+a_{3} u^{2}+a_{4} u^{3}
$$

where $a_{j}, j=1, \ldots, 4$, are deterministic coefficients that are obtained by making the first four central moments of $S_{u}(u)$ equal to those of $x_{s}$. 
After the four parameters $a_{1}, a_{2}, a_{3}$ and $a_{4}$ are determined, the random number of $x$ can be readily obtained with the aid of the following equation using those of a standard normal random variable.

$$
x=\sigma\left(a_{1}+a_{2} u+a_{3} u^{2}+a_{4} u^{3}\right)+\mu
$$

Making the first four central moments of $S_{u}(u)$ equal to those of $x_{s}$, the following equations can be obtained in terms of the parameters $a_{1}, a_{2}, a_{3}$ and $a_{4}$ by simply making use of the first 12 moments of the standard normal random variable $u$ :

$$
\begin{aligned}
\mu_{s u} & =a_{1}+a_{3}=0 \\
\sigma_{s u}^{2} & =a_{2}^{2}+2 a_{3}^{2}+6 a_{2} a_{4}+15 a_{4}^{2}=1 \\
\alpha_{3 s u} & =6 a_{2}^{2} a_{3}+8 a_{3}^{3}+72 a_{2} a_{3} a_{4}+270 a_{3} a_{4}^{2}=\alpha_{3} \\
\alpha_{4 s u} & =3\left(a_{2}^{4}+20 a_{2}^{3} a_{4}+210 a_{2}^{2} a_{4}^{2}+1260 a_{2} a_{4}^{3}\right. \\
& \left.+3465 a_{4}^{4}\right)+12 a_{3}^{2}\left(5 a_{2}^{2}+5 a_{3}^{2}+78 a_{2} a_{4}\right. \\
& \left.+375 a_{4}^{2}\right)=\alpha_{4}
\end{aligned}
$$

where $\alpha_{4 s u}$ and $\alpha_{4}$ are the fourth dimensionless central moments, i.e. the kurtosis of $S_{u}$ and $x$, respectively.

Simplifying Eq. 15, the following equations of parameters $a_{2}$ and $a_{4}$ can be obtained:

$$
\begin{aligned}
& 2 A_{1} A_{2}^{2}=\alpha_{3}^{2} \\
& 3 A_{1} A_{3}+3 A_{4}=\alpha_{4}
\end{aligned}
$$

where

$$
\begin{aligned}
A_{1}= & 1-a_{2}^{2}-6 a_{2} a_{4}-15 a_{4}^{2} \\
A_{2}= & +a_{2}^{2}+24 a_{2} a_{4}+105 a_{4}^{2} \\
A_{3}= & +5 a_{2}^{2}+126 a_{2} a_{4}+675 a_{4}^{2} \\
A_{4}= & a_{2}^{4}+20 a_{2}^{3} a_{4}+210 a_{2}^{2} a_{4}^{2} \\
& +1260 a_{2} a_{4}^{3}+3465 a_{4}^{4}
\end{aligned}
$$

After parameters $a_{2}$ and $a_{4}$ have been obtained from Eq. 16 , parameters $a_{1}$ and $a_{3}$ can be readily given as

$$
a_{3}=-a_{1}=\frac{\alpha_{3}}{2 A_{2}}
$$

From Eq. 16a, one can see that the values of $a_{2}$ and $a_{4}$ are independent of the sign of $\alpha_{3}$. For specified values of $\alpha_{3}$ and $\alpha_{4}$, the parameters $a_{2}$ and $a_{4}$ can be obtained from Eq. 16, which can be solved using common subroutines of nonlinear equations such as the "FindRoot" function in "Mathematica" software (Wolfram 1996).

In particular, if $\alpha_{3}=0$ and $\alpha_{4}=3$, then the parameters are obtained as $a_{1}=a_{3}=a_{4}=0, a_{2}=1$, and Eq. 13 will degenerate as $x_{s}=u$. If $\alpha_{3}=2 \sqrt{2}$ and $\alpha_{4}=15$, the parameters are then obtained as $a_{3}=-a_{1}=\sqrt{2} / 2, a_{2}=a_{4}=0$, and Eq. 13 will degenerate as $x_{s}=-\sqrt{2} / 2+\sqrt{2} u^{2} / 2$. If $\alpha_{3}=0$, $\alpha_{4}=46.2$, the parameters are then obtained as $a_{1}=a_{2}=a_{3}=0, a_{4}=1 / \sqrt{15}$, and Eq. 13 will degenerate as $x_{s}=u^{3} / \sqrt{15}$.

\section{Operable area of Eq.13 in the $\alpha_{3}{ }^{2}-\alpha_{4}$ Plane}

In the present paper, the values of parameters $a_{2}$ and $a_{4}$ are obtained using the "FindRoot" function in "Mathematica" software. For a specified value of $\alpha_{3}$, when the values of $\alpha_{4}$ are below a limit value, the "FindRoot" function becomes inoperable. Using the limit values of $\alpha_{4}$ for which Eq. 16 is inoperable corresponding to the selected $\alpha_{3}$, a lower boundary line in the $\alpha_{3}{ }^{2}-\alpha_{4}$ plane can be depicted as shown in Fig. 1, in which the operable area of Eq. 13 is indicated by the shaded region. The lower boundary line for which Eq. 13 is operable is found to be a straight line approximately expressed by

$$
\alpha_{4}=1.88+1.55 \alpha_{3}^{2}
$$

The variations of parameters $a_{2}$ and $a_{4}$ with respect to a large range of $\alpha_{4}$ values are shown in Fig. $2 \& 3$, in which $\alpha_{3}$ is assumed to be $0,1,2$ and 3 . Figures $2 \& 3$ show that even for very large values of $\alpha_{4}$, continuous values of parameters $a_{2}$ and $a_{4}$ can also be obtained using Eq. 16. That is to say, the proposed transformation is operable for all values above the boundary line shown in Fig. 1.

In Fig. 1, the limit for all distributions expressed as $\alpha_{4}=\alpha_{3}^{2}+1$ (Johnson \& Kotz 1970) is also depicted along with $\alpha_{3}{ }^{2}-\alpha_{4}$ relationship for some commonly used distributions, i.e., lognormal, Weibull, Gamma, Normal, Laplace, Exponential, Gumbel and Rayleigh distributions. One can see that the operable area of Eq. 13 covers a large area in the $\alpha_{3}^{2}-\alpha_{4}$ plane, and the $\alpha_{3}{ }^{2}$ $\alpha_{4}$ relationship for most commonly used distributions are in the operable area of Eq. 13. This implies that Eq. 13 is generally operable for common engineering use.

\section{Numerical Examples and Investigations}

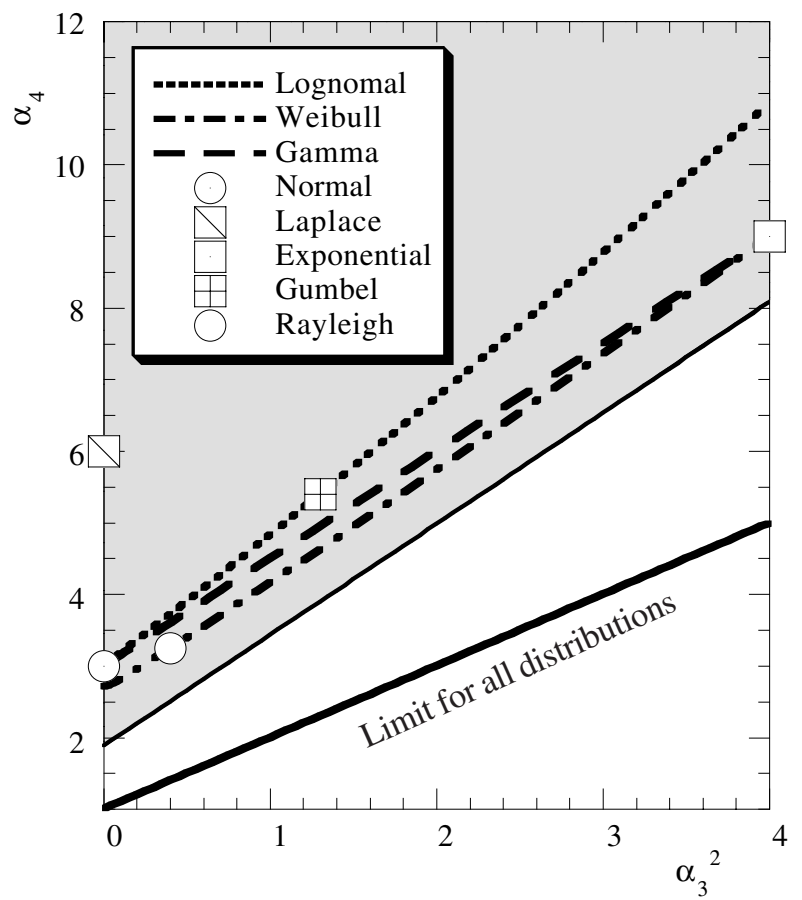

Fig. 1. Operable area of Eq.13 


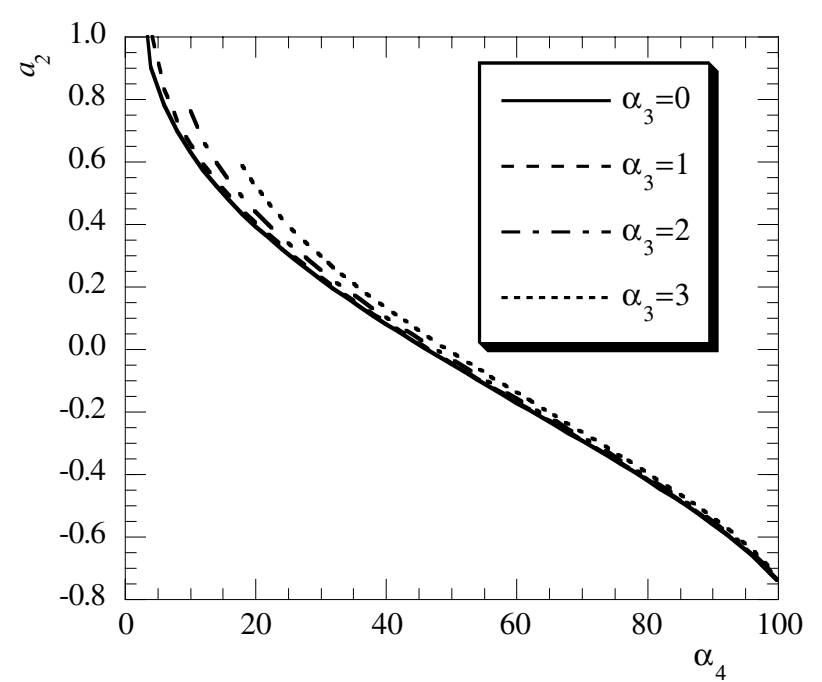

Fig. 2. Variation of parameter $a_{2}$ with respect to $a_{4}$

\section{Example 1: Random Number Generation for Some Commonly Used Random Variables}

In order to investigate the efficiency of the proposed method, six commonly used random variables that have known CDFs are selected in the first example. The first four moments of the six distributions are listed in Table 1, and using the first four moments and Eq. 16, the four parameters $a_{1}, a_{2}, a_{3}$ and $a_{4}$ in Eq. 14 are obtained in Table 1. After the parameters $a_{1}, a_{2}, a_{3}$ and $a_{4}$ are obtained, the random numbers of the six distributions can be easily generated using Eq. 14 with the aid of the random numbers of $u$. The histograms obtained using Eq. 14 for the six distributions are shown in Fig. 4, in which the thick solid lines indicate the PDF exactly obtained from the six distributions. In Fig. 4, the number of samplings is taken to be 40,000 for all the cases, and the histograms have be scaled using the following equation in order to compare with the exact PDF.

$$
h_{i}=H_{i} / \Delta x
$$

where $H_{i}$ is the relative frequency and $\Delta x$ is the category interval of the histogram. $h_{i}$ is the scaled relative frequency.

From Fig. 4, one can see that the histograms obtained using Eq. 14 fit the exact PDF very well for all

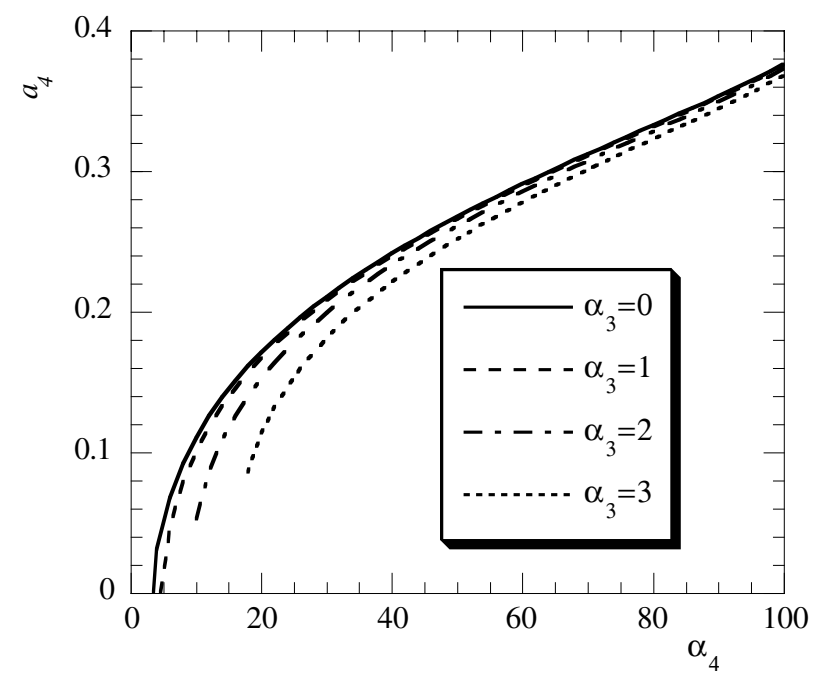

Fig. 3. Variation of parameter $a_{4}$ with respect to $a_{4}$

six cases. This implies that the random number generation method using Eq. 14 is suitable.

According to Eqs. 1 and 14, the percentile point of a distribution with first four moments corresponding to a percentage value $\alpha$ can be explicitly evaluated as

$$
x_{\alpha}=\sigma\left(a_{1}+a_{2} u_{\alpha}+a_{3} u_{\alpha}^{2}+a_{4} u_{\alpha}^{3}\right)+\mu
$$

where $u_{\alpha}$ is the percentile point of a standard normal random variable.

The percentile points of $1 \%, 5 \%, 10 \%, 25 \%, 50 \%$, $75 \%, 90 \%, 95 \%$ and $99 \%$ evaluated using Eq. 19 for the six distributions are listed in Table 2 along with the exact results obtained directly using the CDFs of the six distributions. Table 2 reveals that although only the first four moments are used in Eq. 14, it provides good approximations of the exact percentile points for all the percent levels and all the six cases.

In order to investigate the efficiency of the method for the case that only the first three moments are known, the random numbers of the above six commonly used random variables are also generated using their first three moments only. For samplings in which the number is taken to be 40,000 , the histograms obtained using Eq. 7 with the aid of the random numbers of $u$ for the six cases are shown in Fig. 5, in which the thick solid lines indicate the PDF which is exactly obtained from the six distributions. From the comparison

Table 1. Corresponding distributions and parameters of Fig.4

\begin{tabular}{|l|l|c|c|c|c|c|c|c|c|}
\hline Case & \multirow{2}{*}{ Distribution } & \multicolumn{4}{|c|}{ moments } & \multicolumn{4}{c|}{ parameters } \\
\cline { 3 - 9 } & & mean & COV & $\alpha_{3}$ & $\alpha_{4}$ & $a_{1}$ & $a_{2}$ & $a_{3}$ & $a_{4}$ \\
\hline (a) & Gamma & 100 & 0.3 & 0.6 & 3.54 & -0.0992 & 0.9827 & 0.0992 & 0.00245 \\
(b) & Lognormal & 100 & 0.3 & 0.927 & 4.5659 & -0.1426 & 0.9308 & 0.1426 & 0.01594 \\
(c) & Weibull & 100 & 0.2 & -0.3519 & 3.0039 & 0.0614 & 1.0178 & -0.0614 & -0.00726 \\
(d) & Weibull & 100 & 0.6 & 0.8496 & 3.7320 & -0.1543 & 1.0105 & 0.1543 & -0.01167 \\
(e) & Gumbel & 100 & 0.4 & 1.1396 & 5.4 & -0.1683 & 0.8969 & 0.1683 & 0.02418 \\
(f) & Rayleigh & 100 & 0.523 & 0.6311 & 3.2451 & -0.1156 & 1.0282 & 0.1156 & -0.01407 \\
\hline
\end{tabular}




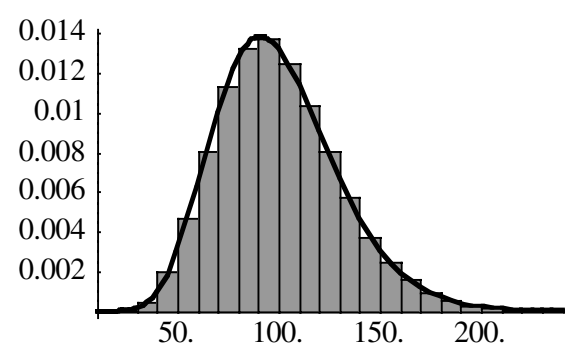

(a) Gamma

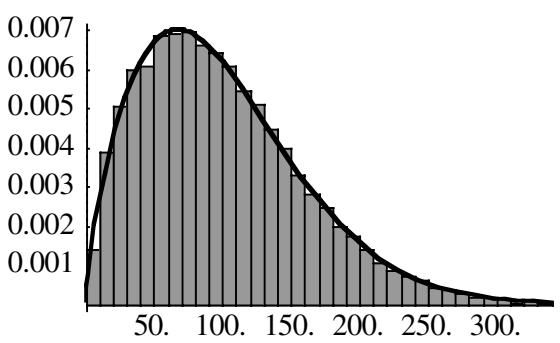

(d) Weibull $(\mathrm{COV}=0.6)$

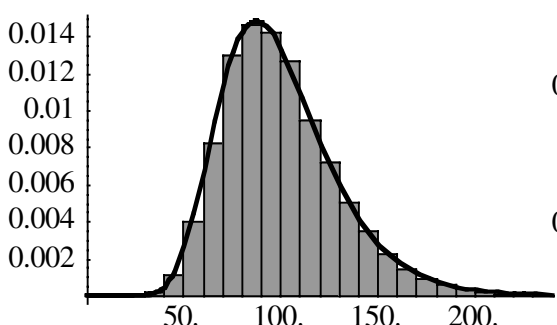

(b) Lognormal

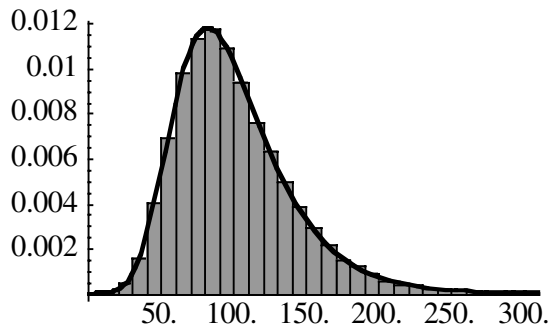

(e) Gumbel

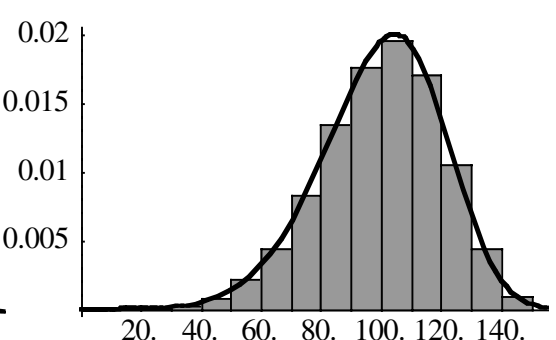

(c) Weibull $(\mathrm{COV}=0.2)$

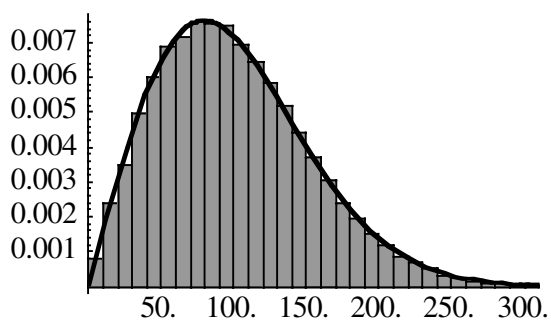

(f) Rayleigh

Fig. 4. Random number generation for some common used distributions using the first four moments

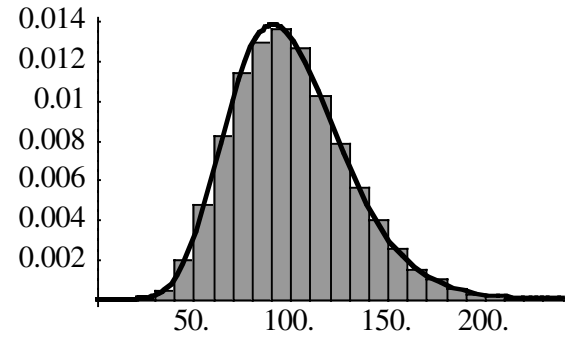

(a) Gamma

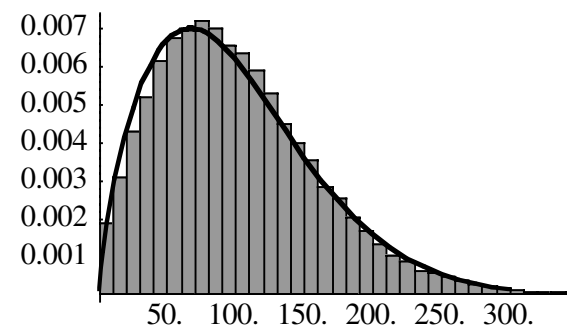

(d) Weibull $(\mathrm{COV}=0.6)$

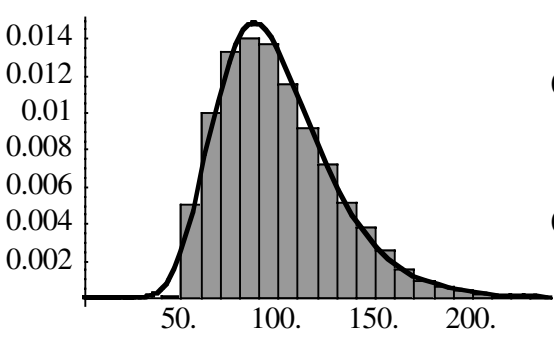

(b) Lognormal

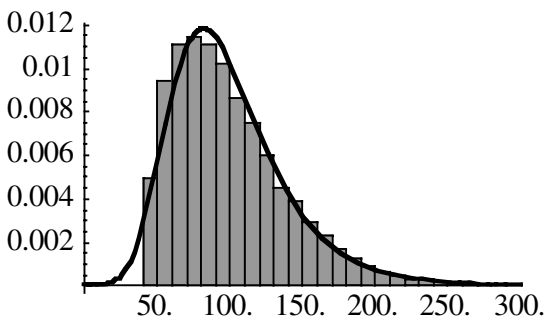

(e) Gumbel

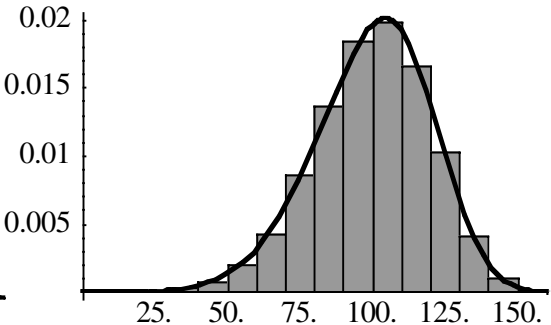

(c) Weibull $(\mathrm{COV}=0.2)$

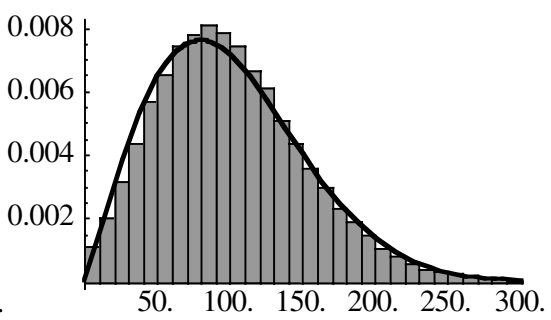

(f) Rayleigh

Fig. 5. Random number generation for some common used distributions using the first three moments

between Figs. $4 \& 5$, one can see that although the accuracy of fit using Eq. 7 is generally not as good as that using Eq. 14, the histograms obtained using Eq. 7 generally fit the exact PDF for all the six distributions. Considering the fact that the information of first three moments only are used in Eq. 7 while the information of the first four moments are used in Eq. 14, one can see that Eq. 7 can be used to generate the random numbers of a random variable in the case that only the first three moments are known.

The percentile points of $1 \%, 5 \%, 10 \%, 25 \%, 50 \%$,
$75 \%, 90 \%, 95 \%$ and $99 \%$ evaluated using Eq. 7 for the six cases are listed in Table 3 along with the exact results obtained directly using the CDFs of the six distributions. Table 3 reveals that although only the first three moments are used in Eq. 7, it provides good approximations of the exact percentile points for all the six cases at all the percent levels that are larger than $5 \%$. For cases $\mathrm{b}, \mathrm{d}$, e and $\mathrm{f}$ at percent level $1 \%$, the percentile points obtained from Eq. 7 have significant errors since only the first three moments are used. Therefore, Eq. 7 is only recommended for cases in 
Table 2. Percentile Point Evaluation for the Six Distributions Using the First Four Moments

\begin{tabular}{|c|c|c|c|c|c|c|c|c|c|c|c|c|}
\hline \multirow{2}{*}{$\%$} & \multicolumn{2}{|c|}{ Gamma(a) } & \multicolumn{2}{c|}{ Lognormal(b) } & \multicolumn{2}{c|}{ Weibull(c) } & \multicolumn{2}{c|}{ Weibull(d) } & \multicolumn{2}{c|}{ Gumbel(e) } & \multicolumn{2}{c|}{ Rayleigh(f) } \\
\cline { 2 - 13 } & Eq.14 & exact & Eq.14 & exact & Eq.14 & exact & Eq.14 & exact & Eq.14 & exact & Eq.14 & exact \\
\hline 1 & 43.62 & 43.59 & 47.90 & 48.38 & 49.05 & 48.84 & 8.630 & 7.697 & 34.05 & 34.37 & 10.88 & 11.31 \\
5 & 56.25 & 56.27 & 59.24 & 59.10 & 65.07 & 64.70 & 19.18 & 19.89 & 48.16 & 47.78 & 25.17 & 25.56 \\
10 & 63.97 & 63.99 & 65.95 & 65.75 & 73.43 & 73.25 & 29.72 & 30.24 & 56.31 & 55.99 & 36.55 & 36.63 \\
25 & 78.47 & 78.48 & 78.69 & 78.58 & 86.98 & 87.11 & 54.27 & 54.29 & 71.84 & 71.81 & 60.68 & 60.52 \\
50 & 97.02 & 97.02 & 95.72 & 95.78 & 101.2 & 101.4 & 90.74 & 90.59 & 93.27 & 93.43 & 93.96 & 93.94 \\
75 & 118.3 & 118.3 & 116.6 & 116.8 & 114.4 & 114.3 & 135.6 & 135.7 & 120.8 & 120.9 & 132.7 & 132.9 \\
90 & 139.8 & 139.9 & 139.5 & 139.5 & 125.0 & 124.7 & 182.2 & 182.3 & 152.3 & 152.2 & 171.2 & 171.2 \\
95 & 153.9 & 153.9 & 155.4 & 155.2 & 130.7 & 130.5 & 212.4 & 212.5 & 174.8 & 174.6 & 195.4 & 195.3 \\
99 & 182.6 & 182.7 & 189.9 & 189.6 & 140.1 & 140.5 & 273.1 & 272.9 & 225.3 & 225.5 & 242.4 & 242.1 \\
\hline
\end{tabular}

Table 3. Percentile Point Evaluation for the Six Distributions Using the First Three Moments

\begin{tabular}{|c|c|c|c|c|c|c|c|c|c|c|c|c|}
\hline$c$ & \multicolumn{2}{|c|}{ Gamma(a) } & \multicolumn{2}{|c|}{ Lognormal(b) } & \multicolumn{2}{|c|}{ Weibull(c) } & \multicolumn{2}{|c|}{ Weibull(d) } & \multicolumn{2}{|c|}{ Gumbel(e) } & \multicolumn{2}{|c|}{ Rayleigh(f) } \\
\hline & Eq.7 & exact & Eq.7 & exact & Eq.7 & exact & Eq.7 & exact & Eq.7 & exact & Eq.7 & exact \\
\hline 1 & & & & 48.3 & 8.45 & & & & & & 1 & 11.31 \\
\hline 5 & & & & & & & & & & & & \\
\hline 10 & 3.89 & 63. & 65.54 & 65.7 & 73.70 & 73.2 & 30.24 & 30.24 & 5.73 & & 37.33 & 36.63 \\
\hline 2 & & & 77.7 & & & 87 & & & & & 62. & 60.52 \\
\hline 50 & 6.98 & 97. & 95.2 & 95. & & & & & & & 94.46 & 93.94 \\
\hline 7. & & 118 & 117 & & & & & & & & 131.9 & 132.9 \\
\hline & & & & & & & & & & & & 171.2 \\
\hline & & & & & & & & & & & & 195.3 \\
\hline 99 & 182.4 & 182.7 & 188.8 & 189.6 & 141.2 & 140.5 & 274.7 & 272.9 & 223.8 & 225.5 & 244.7 & 242.1 \\
\hline
\end{tabular}

Table 4. Corresponding moments and parameters of Fig.6

\begin{tabular}{|l|c|c|c|c|c|c|c|c|}
\hline \multirow{2}{*}{ Case } & \multicolumn{4}{|c|}{ moments } & \multicolumn{4}{c|}{ parameters } \\
\cline { 2 - 9 } & mean & COV & $\alpha_{3}$ & $\alpha_{4}$ & $a_{1}$ & $a_{2}$ & $a_{3}$ & $a_{4}$ \\
\hline 1 & 350 & 0.333 & -0.1721 & 3.2081 & 0.0276 & 0.9792 & -0.0276 & 0.00663 \\
2 & 350 & 0.333 & 0.3456 & 3.3443 & -0.0553 & 0.9749 & 0.0553 & 0.00728 \\
3 & 350 & 0.223 & -0.4642 & 3.8460 & 0.0691 & 0.9347 & -0.0691 & 0.01978 \\
4 & 350 & 0.223 & -0.7608 & 4.1653 & 0.1172 & 0.9408 & -0.1172 & 0.01488 \\
\hline
\end{tabular}

which the information of the first three moments only is known. When the information of the first four moments is known, Eq. 14 should be used.

\section{Example 2: Standardization for A Simple Performance Function}

The second example considers the following simple function of two random variables, which is an elementary reliability model that has several applications

$$
X=R-S
$$

where $R$ is a resistance having $\mu_{R}=500, S$ is a load having $\mu_{S}=150$.
The following four cases are investigated under the assumption that $R$ and $S$ follow different probability distributions.

Case-1: $R$ is Normal and $S$ is Lognormal with $V_{R}=0.2$ and $V_{S}=0.4$.

Case-2: $R$ is Lognormal and $S$ is Weibull with $V_{R}=0.2$ and $V_{S}=0.4$.

Case-3: $R$ is Gamma and $S$ is Gumbel with $V_{R}=0.1$ and $V_{s}=0.4$.

Case-4: $R$ is Weibull and $S$ is Lognormal with $V_{R}=0.1$ and $V_{S}=0.4$.

Using the first four moments of $R$ and $S$, the first four moments of $X$ can be easily obtained in Table 4. Using 


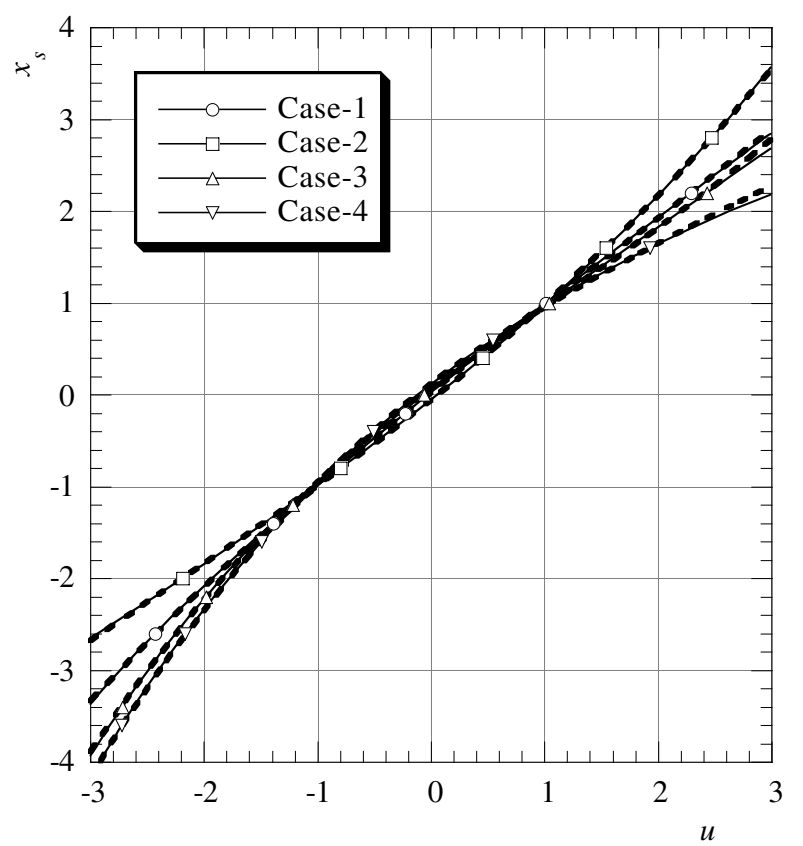

Fig. 6. $u-x$ transformations for Example 2

the first four moments and Eq. 16, the four parameters $a_{1}, a_{2}, a_{3}$ and $a_{4}$ in Eq. 14 are obtained in Table 4. After the parameters $a_{1}, a_{2}, a_{3}$ and $a_{4}$ are obtained, the $u-x$ transformation of $X$ can be easily obtained using Eq. 13. For comparison, the exact transformation is obtained using the PDF/CDFs of $R$ and $S$, which is expressed as:

$$
F_{X}\left(x_{s}\right)=\int F_{R}\left(\sigma_{x} x_{s}+\mu_{x}+t\right) f_{s}(t) d t
$$

where $F_{X}$ and $F_{R}$ are the CDFs of $X$ and $R$ respectively, $f_{S}$ is the PDF of $S$, and $\sigma_{x}$ and $\mu_{x}$ are the standard deviation and mean value, respectively, of $X$.

The variations of $x$ with respect to $u$ obtained using Eq. 13 are shown in Fig. 6 along with those exactly obtained using Eq. 22, where the thick dash lines indicate the results of Eq.13 and the thin solid lines indicate the exact results. Figure 6 shows that Eq. 13 provides good approximations of the exact results.

Using the first four moments of $R$ and $S$, the random number of $R$ and $S$ can be easily generated with the aid of Eq. 14, and the MCS results of the probability of failure are obtained as $3.01 \times 10^{-3}(\beta=2.747), 2.8 \times 10^{-4}$ ( $\beta=3.450), 5.9 \times 10^{-4}(\beta=3.244), 8.4 \times 10^{-4}(\beta=3.142)$ for the four cases, when the number of samplings is taken to be 100,000 . The MCS results using the CDFs with the same number of samplings are obtained as $3.11 \times 10^{-3}(\beta=2.736), 3.0 \times 10^{-4}(\beta=3.432), 6.0 \times 10^{-}$ ${ }^{4}(\beta=3.239), 8.5 \times 10^{-4}(\beta=3.138)$. One can see that the results using the first four moments are in good agreement with those using the CDFs for all the four cases.

\section{Example 3: Data Fitting for Random Variables with Unknown CDFs}

According to Eqs. 1 and 14, a four parameter distribution can be defined by the following probability density function (PDF) of $x$.

$$
f(x)=\frac{\phi(u)}{\sigma\left(a_{2}+2 a_{3} u+3 a_{4} u^{2}\right)}
$$

where $\phi$ is the PDF of $u$.

For a random variable with unknown $\mathrm{CDF} / \mathrm{PDF}$, although the MCS in the present paper has not used its $\mathrm{CDF} / \mathrm{PDF}$, the use of Eq.14 essentially implies that the variable obeys a distribution defined by Eq. 23 . In order to investigate the efficiency of Eq. 23 in fitting statistical data of a random variable with unknown CDF/PDF, the third example uses the practical data of H-shape structural steel collected by Ono et. al(1986). The fitting result of the histogram of the ratio between measured values and nominal values for the thickness is shown in Fig. 7, in which the number of data is 885 and the first-four moments of the data are obtained as $\mu=0.986, \sigma=0.0457, \alpha_{3}=0.883, \alpha_{4}=5.991$. In Fig. 7, the PDFs of the normal and lognormal distributions whose mean values and deviations are equal to those of the data, the PDF of the three-parameter distribution Zhao

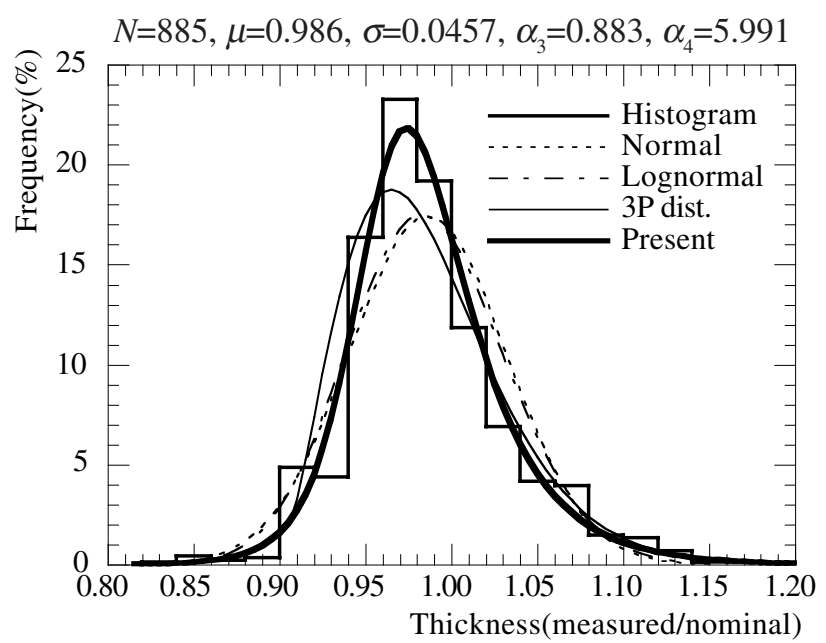

Fig. 7. Data Fitting for Thickness

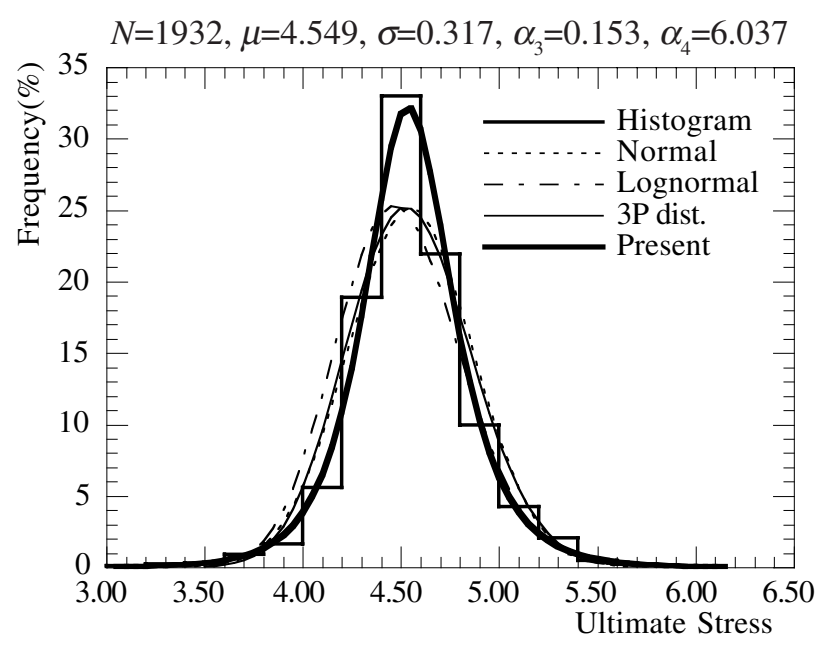

Fig. 8. Data Fitting for Ultimate Stress 
et. al.(2001) whose first-three moments are equal to those of the data, and the PDF of the four-parameter distribution described in Eq. 23 whose first-four moments are equal to those of the data, are depicted. Figure 7 reveals the following:

(1) The PDFs of the normal distribution and lognormal distribution have the greatest differences from the histogram of the statistical data among the four distributions. Since normal distribution is a symmetrical distribution ( $\left.\alpha_{3}=0, \alpha_{3}=3\right)$, it obviously can not be used to fit the histogram that has such a large skewness $\left(\alpha_{3}=0.883\right)$ and kurtosis $\left(\alpha_{4}=5.991\right)$. Although the lognormal distribution can reflect skewness and kurtosis in some degree, the skewness and kurtosis are dependent on the coefficient of variation. Since the coefficient of variation for this data is very small (0.0463), the skewness and kurtosis of lognormal distribution corresponding to this coefficient of variation are too small ( $\left.\alpha_{3}=0.139, \alpha_{4}=3.0344\right)$ to match those of the data.

(2) Since the skewness of the three-parameter distribution is equal to that of the statistical data, it fits the histogram much better than the normal and lognormal distributions. However, the kurtosis of the three-parameter distribution is dependent on the skewness. The kurtosis corresponding the skewness of this data is obtained as $\alpha_{4}=4.04$ which is obviously too small to match that of the data. Therefore, the PDF of the three-parameter distribution is also very different from the histogram of the statistical data.

(3) Since both the skewness and kurtosis of the fourparameter distribution are equal to those of the statistical data, it fits the histogram much better than the normal, lognormal and the three-parameter distributions. That is to say, the four-parameter distribution is more suitable for this statistical data.

Similarly, the fitting result of the histogram of the ultimate stress is shown in Fig. 8, in which the number of data is 1932 and the first-four moments of the data are obtained as $\mu=4.549, \sigma=0.317, \alpha_{3}=0.153, \alpha_{4}=6.037$. From Fig. 8, one can see that since the skewness for this data is quite small, the three-parameter distribution can not show significant improvement upon the normal and lognormal distributions. One can also see that since both the skewness and kurtosis of the four-parameter distribution are equal to those of the statistical data, it fits the histogram much better than the normal, lognormal and the three parameter distributions. That is to say, the four-parameter distribution is more suitable for this statistical data.

\section{Example 4: Reliability Analysis of A Column}

The fourth example considers the following simple performance function, a simple compressive limit state of a structural column.

$$
G(\mathbf{X})=A x_{1} x_{2}-x_{3}
$$

Where $A$ is the nominal section area, $x_{1}$ is a random variable presenting the uncertainty included in $A, x_{2}$ is yield stress and $x_{3}$ is a compressive load. The column is made of H-shape structural steel with a section area of
$A=72.38 \mathrm{~cm}^{2}$. The CDFs of $x_{1}$ and $x_{2}$ are unknown, the only information about them are their first four moments (Ono, Idota \& Kawahara 1986), i.e., $\mu_{1}=0.990$, $\sigma_{1}=0.051, \alpha_{31}=0.709, \alpha_{41}=3.692, \mu_{2}=3.055 \mathrm{t} / \mathrm{cm}^{2}$, $\sigma_{2}=0.364, \alpha_{32}=0.512, \alpha_{42}=3.957 . x_{3}$ is assumed as a lognormal variable with mean value $\mu_{3}=100 t$ and standard deviation $\sigma_{4}=40$.

Although the CDFs of $x_{1}$ and $x_{2}$ are unknown, using Eq.14, the random sampling of $x_{1}$ and $x_{2}$ can be easily generated without using their CDFs, and the MCS can thus be easily conducted. The probability of failure is obtained as $P_{f}=0.0188(\beta=2.079)$ when the number of samplings is taken to be 10,000 .

\section{Conclusion}

In order to include the random variables with unknown CDFs into MCS, an inverse normal transformation is suggested. Using the proposed method, the random numbers of random variables with an unknown CDF can be easily generated utilizing those of a standard normal random variable. Through some examples, the efficiency of the method is investigated. It is found that although the method is quite simple, it is accurate enough to include the random variables with unknown CDF into MCS of structural reliability.

\section{References}

1) Ang, A. H-S. and Cornell, C.A. 1974. Reliability basis of structural safety and design. J. Struct. Engrg., ASCE, 100(9), 17551769.

2) Ang, A. H-S. and Tang, W.H. 1984. Probability Concepts in Engineering Planning and Design, Vol. II: Decision, Resik, and Reliability, J. Wiley \& Sons.

3) Der Kiureghian, A. and Liu, P.L. 1986. Structural reliability under incomplete probability information. J. Engrg. Mech., ASCE, 112(1), 85-104.

4) Der Kiureghian, A. 1989. Measures of structural safety under imperfect states of knowledge. J. Struct. Engrg., ASCE, 115(5), 1119-1140.

5) Johnson, N.L. and Kotz, S. 1970, Continuous univariate distributions-1, John Wiley \& Sons, New York, 9-35.

6) Ono, T., Idota, H. and Kawahara, H. 1986. A statistical study on resistances of steel columns and beams using higher order moments. J. Struct. and constr. engrg., AIJ, No. 370, 19-27. (in Japanese).

7) Ono, T. and Idota, H. 1986. Proposal of the high order moment standardizing method and definition of reliability index. J. Struct. and constr. engrg., AIJ, No. 359, 43-49 (in Japanese).

8) Wolfram S. 1996, Mathematica, a system for doing mathematics by computer, 2nd edition, Seiunsya, Tokyo. (Japanese translation).

9) Zhao, Y.G. and Ono, T. 2000. Third-moment standardization for structural reliability analysis. J. Struct. Engrg., ASCE, 126(6), 724-732.

10) Zhao, Y.G., Ono, T. Idota, H. and Hirano, T., 2001, A three-parameter distribution used for structural reliability evaluation, $J$. Struct. and constr. engrg., AIJ, No.546, 31-38. 